ANNALES

POLONICI MATHEMATICI

XLVIII (1988)

\title{
Directional qualitative cluster sets
}

\author{
by A. K. LayeK (Calcutta)
}

\begin{abstract}
For arbitrary function $f$ defined on the half plane $H$ above the real line $R$, the interesection properties between directional and sectorial qualitative cluster sets $C_{q}(f, x, \theta)$ and $C_{q}(f, x, S)$, and between directional cluster set $C(f, x, \theta)$ and directional qualitative cluster set $C_{q}(f, x, \theta)$ are studied. The results proved here state that: If $f: H \rightarrow W, W$ is a compact, normal and second countable topological space, then (i) except a countable set of points on $R$, for every sector $S$ in $H, C_{q}(f, x, S)$ intersects $C_{q}(f, x, \theta)$ for a residual set of $\theta$ in $(0, \pi)$, (ii) except a first calegory set on $R$, for fixed $\theta$ in $(0, \pi), C(f, x, \theta)$ intersects $C_{q}(f, x, \varphi)$ for each $\varphi(0, \pi)$, and (iii) except a countable set on $R$, for every $\varphi$ in $(0, \pi), C_{q}(f, x, \varphi)$ intersects $C(f, x, \theta)$ for a residual set of $\theta$ in $(0, \pi)$.
\end{abstract}

1. Let $R$ be the real line and $H=R \times(0, \alpha)$. Let $L_{\theta}(x)$ be a ray in $H$ emanating from $x$ in $R$ and in the direction $\theta, 0<\theta<\pi$. Also, let $S_{\alpha \beta}$ denote a sector in $H$ having vertex at the origin, defined by

$$
S_{\alpha \beta}=\{Z: Z \in H: 0<\alpha<\arg (z)<\beta<\pi\} .
$$

$S_{\alpha \beta}(x)$ is the translate of $S_{\alpha \beta}$, obtained by taking the origin at $x$. If there is no confusion, simply $S$ and $S(x)$ will stand to denote $S_{\alpha \beta}$ and $S_{\alpha \beta}(x)$. For $x \in R$ and $r>0$, set

$$
\begin{aligned}
K(x, r) & =\{Z: Z \in H:|Z-x|<r\}, \\
S(x, r) & =S(x) \cap K(x, r),
\end{aligned}
$$

and

$$
L_{\theta}(x, r)=L_{\theta}(x) \cap K(x, r) .
$$

Further, $\bar{E}$ will denote the closure of the set $E$.

Throughout the paper, f.c. and s.c. will mean first category and second category, respectively.

If $f: H \rightarrow W, W$ is a topological space, the qualitative cluster set $C_{q}(f, x)$ of $f$ at $x$ is the set of all $w$ in $W$ for which $f^{-1}(u) \cap K(x, r)$ is s.c. for all $r>0$ and for every open set $u$ in $W$ containing $w$. The definitions of sectorial qualitative cluster set $C_{q}(f, x, S)$ and directional qualitative cluster set $C_{q}(f, x, \theta)$ are similar with $K(x, r)$ replaced by $S(x, r)$ and $L_{\theta}(x, r)$, respect- 
ively. The cluster set $C(f, x, S)$ (resp. $C(f, x, \theta)$ ) of $f$ at $x$ in $S$ (in the direction $\theta$ ) is the set of all $w$ in $W$ for which $x \in \overline{f^{-1}(u) \in S(x)}$ $\left(x \in \overline{\left.f^{-1}(u) \cap L_{\theta}(x)\right)}\right.$ for every open set $u$ in $W$ containing $w$.

2. Wilczyński [6] proved that if $f: H \rightarrow R$ has the Baire property and $\theta \in(0, \pi)$ is a fixed direction, then $C_{q}(f, x)=C_{q}(f, x, \theta)$ at all $x$ but a f.c. set on $R$. Supplementing this result, Evans and Humke [1] proved that $C_{q}(f, x)$ $=C_{q}(f, x, \theta)$ for a residual set of directions $\theta$ in $(0, \pi)$ at all $x$ but a f.c. set on $R$. In [3] it is also proved that if $\{S\}$ is the collection of all sectors in $H$ then

$$
\bigcup\left\{C_{q}(f, x, S): S \in\{S\}\right\}=C_{q}(f, x, \theta)
$$

for a residual set of directions $\theta \in(0, \pi)$ at all $x$ but a $\sigma$-porous set [7] on $R$. In [2], intersecting properties of qualitative cluster sets are studied. A result of this paper states that if $f: H \rightarrow W$ is arbitrary, $W$ is a compact, normal and second countable topological space, then except a countable set of points $x$ in $R, C_{q}\left(f, x, S_{1}\right) \cap C_{q}\left(f, x, S_{2}\right) \neq \emptyset$ for each pair of sectors $S_{1}$ and $S_{2}$ in $H$. Here further properties of qualitative cluster sets are studied.

3. In this section, some sets are defined which will be used in the sequel. For $E \subset H$ and $x \in R$ set

$E(x)=\left\{\theta: 0<\theta<\pi: L_{\theta}(x, r) \cap E\right.$ is residual in $L_{\theta}(x, r)$ for some $\left.r>0\right\}$,

$$
\hat{E}(x)=\left\{\theta: 0<\theta<\pi: x \notin \overline{L_{\theta}(x) \cap E}\right\},
$$

and

$$
E[x]=\{S: S \subset H: S(x, r) \cap E \text { is f.c. for some } r>0\} .
$$

For positive integer $n$ and rationals $\alpha, \beta$ in $(0, \pi)$, let

$$
\begin{aligned}
& E_{n}(x)=\left\{\theta: 0<\theta<\pi: L_{\theta}(x, 1 / n) \cap E \text { is residual in } L_{\theta}(x, 1 / n)\right\}, \\
& \hat{E}_{n}(x)=\left\{\theta: 0<\theta<\pi: L_{\theta}(x, 1 / n) \cap E=\emptyset\right\}, \\
& E_{n}[x]=\{S: S \subset H: S(x, 1 / n) \cap E \text { is f.c. }\}, \\
& E_{n}[x, \alpha, \beta]=\left\{S: S \subset H \backslash \bar{S}_{\alpha \beta}, S(x, 1 / n) \cap E \text { is f.c. }\right\}, \\
& \text { and, for any set } \Theta \subset(0, \pi), \text { let }
\end{aligned}
$$

$$
\Theta_{\alpha \beta}=\Theta \cap(\alpha, \beta)
$$

For a set $E \subset H$ we also define

$(E)=\{x: x \in R ; E(x)$ is s.c. and $E[x] \neq \varnothing\}$,

$(E)_{\theta}^{1}=\left\{x: x \in R ; x \notin \overline{L_{\theta}(x) \cap E}\right.$ and $\left.E(x) \neq \emptyset\right\}$,

$(E)^{0}=\{x: x \in R ; \hat{E}(x)$ is s.c. and $E(x) \neq \emptyset\}$.

Lemma 1. If $E \subset H$ is arbitrary, then the set $(E)$ is countable. 
Proof. For positive integers $n$ and $p$ and rationals $\alpha, \beta, 0<\alpha<\beta<\pi$ : let

$$
T_{n \sigma \alpha \beta}=\left\{x: x \in R ; E_{n \alpha \beta}(x) \text { is s.c. and } E_{p}[x, \alpha, \beta] \neq \emptyset\right\} \text {, }
$$

where $E_{n a \beta}(x)=E_{n}(x) \cap(\alpha, \beta)$.

If $x \in(E)$ then $E[x] \neq \emptyset$ and $E(x)$ is s.c. Thus one can choose positive integers $p, n$ and two rationals $\gamma, \delta ; 0<\gamma<\delta<\pi$ such that $S_{y \delta}(x, 1 / p) \cap E$ is f.c. and $E_{n}(x)$ is s.c. Then by a result in [5], p. 56, either $(0, \gamma)$ or $(\delta, \pi)$ contains a point $\theta^{\prime} \in E_{n}(x)$ such that every neighbourhood of $\theta^{\prime}$ intersects $E_{n}(x)$ in a s.c. set. Thus there exist rationals $\alpha, \beta ; 0<\alpha<\beta<\pi$ such that $(\alpha, \beta) \cap E_{n}(x)$ is a s.c. set and $[\alpha, \beta] \cap[\gamma, \delta]=\emptyset$. Hence $E_{n \alpha \beta}(x)$ is a s.c. set and $E_{p}[x, \alpha \beta] \neq \emptyset$. These imply that $x \in T_{n p a \beta}$, and hence $(E)$ is contained in a countable union of sets $T_{n p \alpha \beta}$.

If possible, let $T=T_{n p a \beta}$ be uncountable for some $n, p$ and $\alpha$ and $\beta$. Then there is $x_{0}$ in $T$ which is a two-sided limit point of $T$. Let $S\left(x_{0}\right) \in E_{p}\left[x_{0}, \alpha, \beta\right]$. Then $S\left(x_{0}, 1 / p\right) \cap E$ is a f.c. set. Let $r>0$. Then there is $\eta \in\left(x_{0}-r, x_{0}+r\right)$ such that $S_{\alpha \beta}(\eta, 1 / n)$ intersects $S\left(x_{0}, 1 / p\right)$ in a quadrilateral $Q$ (say), and $E_{n a \beta}(\eta)$ is a s.c. set. Since for $\theta \in E_{n a \beta}(\eta), L_{\theta}(\eta, 1 / n) \cap E$ is residual in $L_{\theta}(\eta, 1 / n)$ and $E_{n \alpha \beta}(\eta)$ is a s.c. set, therefore $Q \cap E$ is a second category set in $H$ [5], p. 56. Thus $S\left(x_{0}, 1 / p\right) \cap E$ is a second category set, a contradiction. Thus $T_{n p a \beta}$ is countable for all positive integers $n, p$ and rationals $\alpha, \beta ; 0<\alpha<\beta<\pi$. Hence $(E)$ is countable.

LEMMA 2. If $E \subset H$ is arbitrary and if $\theta \in(0, \pi)$ is a fixed direction, then the set $(E)^{1}$ is a first category set.

Proof. For positive integers $m$ and $n$, let

$$
P_{m n}=\left\{x: x \in R ; L_{\theta}(x, 1 / m) \cap E=\emptyset \text { and } E_{n}(x) \neq \emptyset\right\} \text {. }
$$

Then $(E)^{1}$ is contained in a countable union of the sets $P_{m n}$.

If possible, let $P_{m n}$ be a s.c. set for some $m$ and $n$. Let $x_{0} \in P_{m n}$ be such that $\left(x_{0}-r, x_{0}\right) \cap P_{m n}$ and $\left(x_{0}, x_{0}+r\right) \cap P_{m n}$ are second category sets for each $r>0$. Let $\theta_{0} \in E_{n}\left(x_{0}\right)$. Then clearly $\theta \neq \theta_{0}$. Let $\eta \in\left(x_{0}-r, x_{0}+r\right) \cap P_{m n}$ be such that $L_{\theta}(\eta, 1 / m) \cap L_{\theta_{0}}\left(x_{0}, 1 / n\right) \neq \emptyset$. Let $\left|x_{0}-\eta\right|=r_{0}$. Set

$$
Z=\left\{z: z \in L_{\theta}(x, 1 / m) \cap L_{\theta_{0}}\left(x_{0}, 1 / n\right) \text { for } x \in\left(x_{0}-r, x_{0}+r\right) \cap P_{m n}\right\} \text {. }
$$

Then $Z \subset H \backslash E$ and $Z$ is a second category set in $L_{\theta_{0}}\left(x_{0}, 1 / n\right)$. This contradicts the fact that $\theta_{0} \in E_{n}\left(x_{0}\right)$. Hence $P_{m n}$ is a first category set for all $m$ and $n$. This proves that $(E)^{1}$ is a first category set.

LEMma 3. If $E \subset H$ is arbitrary, then the set $(E)^{0}$ is countable.

Proof. For rationals $\alpha, \beta, 0<\alpha<\beta<\pi$ and positive integers $m$ and $n$ let

$$
M_{m n \alpha \beta}=\left\{x: x \in R ; \hat{E}_{m \alpha \beta}(x) \text { is s.c. and } E_{n}(x) \backslash[\alpha, \beta] \neq \emptyset\right\}
$$


If $\hat{E}(x)$ is s.c. and $E(x) \neq \emptyset$, then, for some $m$ and $n, \hat{E}_{m}^{\cdot}(x)$ is s.c. set and $E_{n}(x) \neq \emptyset$, i.e., there is some $\theta \in \hat{E}_{m}(x)$ such that $(\theta-\Delta \theta, \theta+\Delta \theta) \cap E_{m}(x)$ is s.c. for each $\Delta \theta>0$ and $E_{n}(x) \neq \emptyset$. If $\Phi \in E_{m}(x)$, then $\theta \neq \Phi$. So there are rationals $\alpha, \beta$ in $(0, \pi)$ such that $\hat{E}_{m \alpha \beta}(x)$ is s.c. and $E_{n}(x) \backslash[\alpha, \beta] \neq \emptyset$. Thus $x \in(E)^{0}$ implies $x \in M_{m \pi \alpha \beta}$ for some $m, n$ and $\alpha, \beta$. Hence $(E)^{0}$ is contained in a countable union of sets $M_{m \text { map }}$.

Suppose $M=M_{\text {max }}$ is uncountable. Let $x_{0} \in M$ be such that, for each $r>0$, $\left(x_{0}-r, x_{0}\right) \cap M$ and $\left(x_{0}, x_{0}+r\right) \cap M$ are non-void. Let $\theta_{0} \in E_{n}\left(x_{0}\right) \backslash[\alpha, \beta]$. Then there is $\eta \in\left(x_{0}-r, x_{0}+r\right)$ such that $\eta \in M$ also, and $S_{a \beta}(\eta, 1 / m) \cap L_{\theta_{0}}\left(x_{0}, 1 / n\right)$ is a segment $J$ (say) with end points at $L_{\alpha}(\eta, 1 / m) \cap L_{\theta_{0}}\left(x_{0}, 1 / n\right)$ and $L_{\beta}(\eta, 1 / m) \cap L_{\theta_{0}}\left(x_{0}, 1 / n\right)$. Since for $\theta \in \hat{E}_{m a \beta}(\eta)$, $L_{\theta}(\eta, 1 / m) \cap E=\varnothing$ and since $\hat{E}_{\operatorname{ma\beta }}(\eta)$ is a second category set in $(\alpha, \beta)$, the set $J \cap(H \backslash E)$ is a second category set in $L_{\theta_{0}}\left(x_{0}, 1 / n\right)$. This contradicts the fact that $\theta_{0} \in E\left(x_{0}\right)$. Thus $M=M_{\text {mna } \beta}$ is countable for all positive integers $m, n$ and rationals $a, \beta, 0<\alpha<\beta<\pi$; and hence the set $(E)^{0}$ is countable.

LEMMA 4. If $f: H \rightarrow W$ is arbitrary, where $W$ is a compact topological space, and if $U$ is an open set containing $C_{q}(f, x, \theta)$, then $L_{\theta}(x, r) \cap f^{-1}(U)$ is residual in $L_{\theta}(x, r)$ for some $r>0$.

The proof follows from Lemma 3 in [2].

LemMa 5. If $f: H \rightarrow W$ is arbitrary, $W$ is a compact topological space, and if $V_{1}$ and $V_{2}$ are closed sets such that $C_{q}(f, x, S) \cap V_{1}=\emptyset$ and $C(f, x, \theta) \cap V_{2}=\emptyset$, then $S(x, r) \cap f^{-1}\left(V_{1}\right)$ is f.c. set for some $r>0$ and $x \notin \overline{L_{\theta}(x) \cap f^{-1}\left(V_{2}\right)}$.

Proof of the first part follows from Lemma 4 in [2] and the proof of the second part is similar.

4. Throughout this section, $W$ is taken to be a compact, normal and second countable topological space.

THEOREM 1. If $f: \dot{H} \rightarrow W$ is arbitrary, then except a countable set of points $x$ on $R$, for every sector $S \subset H$,

$$
C_{q}(f, x, \theta) \cap C_{q}(f, x, S) \neq \emptyset
$$

for a residual set of directions $\theta \in(0, \pi)$.

Proof. Let $I$ be the exceptional set of Theorem 1 in $R$. Let $\mathscr{G}$ be the collection of all sets $G$ which can be expressed as a finite union of members of $B$, where $B=\left\{V_{n}\right\}$ is a countable basis for the topology of $W$. Then $\mathscr{G}$ is a countable collection. For $G \in \mathscr{G}$ let $f^{-1}(\bar{G})=G^{*}$. Let $x_{0} \in I$. Then there are $S\left(x_{0}\right) \subset H$ and a second category set $O\left(x_{0}\right)$ in $(0, \pi)$ such that $C_{q}\left(f, x_{0}, S\right)$ and $C_{q}\left(f, x_{0}, \theta\right)$ are disjoint closed sets for every $\theta \in O\left(x_{0}\right)$. Since $W$ is 
compact and normal, there is $G \in \mathscr{G}$ such that $C_{q}\left(f, x_{0}, S\right) \cap \bar{G}=\emptyset$ and $C_{q}\left(f, x_{0}, \theta\right) \subset G$ for a second category set of directions $\theta \in O\left(x_{0}\right)$. Then by Lemma $5, S\left(x_{0}, r\right) \cap G^{*}$ is a f.c. set for at least one $r>0$, and by Lemma 4 , $L_{\theta}\left(x_{0}, r\right) \cap G^{*}$ is residual for some $r>0$, for a s.c. set of $\theta \in O\left(x_{0}\right)$. Thus the set $G^{*}\left[x_{0}\right] \neq \emptyset$ and $G^{*}\left(x_{0}\right)$ is a s.c. set in $(0, \pi)$. These imply $x_{0} \in\left(G^{*}\right)$ and hence

$$
I \subset \bigcup\left\{\left(G^{*}\right): G \in \mathscr{G}\right\} .
$$

By the result in Lemma $1,\left(G^{*}\right)$ is countable for each $G \in \mathscr{G}$, and hence $I$ is countable. This completes the proof.

TheOREM 1'. If $f: H \rightarrow W$ is arbitrary, then, except a countable set on $R$, at every $x$ in $R$ there is a residual set $O(x) \subset(0, \pi)$ such that for every $\theta \in O(x)$ and every $S \subset H$

$$
C_{q}(f, x, \theta) \cap C_{q}(f, x, S) \neq \emptyset .
$$

This theorem can be proved by considering the collection $\left\{S_{\alpha \beta}\right\}$ of all sectors with rationals $\alpha, \beta$ in $(0, \pi)$ and applying Theorem 1 . (As the proof of Theorem 2 in [4].)

Corollary 1. Let $f: H \rightarrow W$ be arbitrary. Then, except possibly a countable set on $R$, the degeneracy of $C_{q}(f, x, S)$ for any $S$ in $H$ implies that $C_{q}(f, x, \theta)$ have common value for a residual set of directions $\theta \in(0, \pi)$.

(Degeneracy of $C_{q}(f, x, S)$ means that $C_{q}(f, x, S)$ is singleton.)

THEOREM 2. If $f: H \rightarrow W$ is arbitrary and if $\theta \in(0, \pi)$ is a fixed direction, then except a first category set of points $x$ on $R$

$$
C(f, x, \theta) \cap C_{q}(f, x, \Phi) \neq \varnothing
$$

for every $\Phi \in(0, \pi)$.

Proof. Let $K$ be the exceptional set in Theorem 2 . Let $\mathscr{G}$ and $G^{*}$ be the same as in Theorem 1. Let $x_{0} \in K$. Then there is $\theta_{0} \in(0, \pi)$ such that $C(f, x, \theta)$ and $C_{q}\left(f, x_{0}, \theta_{0}\right)$ are disjoint. Let $G \in \mathscr{G}$ be such that $C\left(f, x_{0}, \theta\right) \cap \bar{G}=\emptyset$ and $C_{q}\left(f, x_{0}, \theta_{0}\right) \subset G$. Then by Lemma 4 and Lemma $5, L_{\theta_{0}}\left(x_{0}, r\right) \cap G^{*}$ is residual in $L_{\theta_{0}}\left(x_{0}, r\right)$ for some $r>0$, i.e., $G^{*}\left(x_{0}\right) \neq \emptyset$ and $x_{0} \notin \overline{L_{\theta}\left(x_{0}\right) \cap G^{*}}$. Thus $x_{0} \in\left(G^{*}\right)^{1}$, and consequently it is proved that

$$
K \subset \bigcup\left\{\left(G^{*}\right)^{1}: G \in \mathscr{G}\right) \text {. }
$$

By Lemma $2,\left(G^{*}\right)^{1}$ is a first category set and hence $K$ is a first category set. This completes the proof.

Corollary 2. If $f: H \rightarrow W$ is arbitrary and if $\theta \in(0, \pi)$ is a fixed 
direction, then except a first category set of points $x$ in $R$, the degeneracy of $C(f, x, \theta)$ implies that

$$
\bigcap_{0<\Phi<\pi} C_{q}(f, x, \Phi) \neq \emptyset
$$

Now we give an example to ensure that the exceptional set of Theorem 2 need not be of measure zero.

Example. Let $P \subset R$ be any set of the first category but of positive measure. Set

$$
F=\bigcup\left\{L_{\pi / 2}(x): x \in P\right\} \text {. }
$$

Let $f$ be the characteristic function of $F$. Then clearly for $x \in P, C_{q}(f, x, \theta)$ $=\{0\}$ for every $\theta \in(0, \pi) \backslash\{\pi / 2\}$ and $C(f, x, \pi / 2)=\{1\}$. Hence at every $x \in P, C_{q}(f, x, \theta)$ and $C(f, x, \pi / 2)$ are disjoint for every $\theta \in(0, \pi) \backslash\{\pi / 2\}$

THeOREM 3. If $f: H \rightarrow W$ is arbitrary, then except a countable set of points $x$ on $R$ for each $\Phi \in(0, \pi)$

$$
C(f, x, \theta) \cap C_{q}(f, x, \Phi) \neq \varnothing
$$

for a residual set of direction $\theta \in(0, \pi)$.

Proof. If $T$ be the exceptional set of Theorem 3, then by using Lemma 4 and Lemma 5 as in Theorem 1 and Theorem 2, it can be shown that

$$
T \subset \bigcup\left\{\left(G^{*}\right)^{0}: G \in \mathscr{G}\right\} \text {. }
$$

By Lemma $3,\left(G^{*}\right)^{0}$ is countable and hence $T$ is countable. This completes the proof.

CoROllary 3. If $f: H \rightarrow W$ is arbitrary, then except a countable set of points $x$ on $R$ for each sector $S \subset H$ and each $\theta \in(0, \pi)$

$$
C(f, x, S) \cap C_{q}(f, x, \theta) \neq \varnothing .
$$

Proof. For each $S \subset H$ there are $\alpha^{\prime}$ and $\beta^{\prime}$ in $(0, \pi)$ such that $S=S_{\alpha^{\prime} \beta^{\prime}}$. And for each $\theta \in\left(\alpha^{\prime}, \beta^{\prime}\right), C(f, x, \theta) \subset C(f, x, S)$. Thus the proof is complete by Theorem 3 .

COROllary 4. If $f: H \rightarrow W$ is arbitrary, then, except a countable set of points $x$ in $R$, the degeneracy of $C_{q}(f, x, \theta)$ for any $\theta \in(0, \pi)$ implies that the sets $C(f, x, \theta)$ have a common value for a residual set of directions $\theta \in(0, \pi)$.

\section{References}

[1] M. J. Evans and P. D. Humke, On qualitative cluster sets, Colloq. Math. 37 (1977), 255261.

[2] A. K. Layek, Intersection of qualitative cluster sets, Real Analysis Exchange Vol. 5, No. 2 (1979-1980). 
[3] A. K. Layek On sectorial qualitative cluster sets and directional qualitative cluster sets (to appear).

[4] -, On Disjoint essential cluster sets, Rev. Roum. Math. Pures Appl. 29 (1984), 707-714.

[5] J. C. Oxt oby, Measure and category, Springer Verlag, New York-Heidelberg-Berlin 1971.

[6] W. Wilczyński, Qualitative Cluster Sets, Colloq. Math. 32 (1974), 113-118.

[7] L. Zajicek, Sets of $\sigma$-porosity and sets of $\sigma$-porosity (q), Casopis Pest. Mat. 101 (1976), 350359.

BANGABASI COLLEGE OF COMMERCE, CALCUTTA

WEST BENGAL INDIA

Reçu par la Rédaction le 1985.04.01 